http://dx.doi.org/10.18359/ravi.2241

\title{
La educación a distancia como respuesta a las necesidades educativas del siglo XXI ${ }^{1}$
}

\author{
Anívar Chaves Torres ${ }^{2}$
}

Universidad Nacional Abierta y a Distancia, UNAD

Recibido, septiembre 16 de 2016

Concepto evaluación, noviembre 15 de 2016

Aceptado, diciembre 27 de 2016
Referencia: Chaves Torres, A. (2017). "La educación a distancia como respuesta a las necesidades educativas del siglo XXI”. Revista Academia y Virtualidad, 10, (1), 23-41

\section{Resumen}

El desarrollo tecnológico, especialmente en el área de la información y la comunicación, ofrece a la sociedad herramientas útiles en diferentes campos, entre ellos el de la educación. Por otra parte, la sociedad del conocimiento plantea nuevos retos a los sistemas educativos y a las personas que deben continuar un proceso de formación vitalicio. En este contexto, la educación a distancia se erige como una respuesta decisiva viable ante la extensa demanda de educación, particularmente por personas que no pueden acceder a la formación presencial. No obstante, es poco lo que se conoce sobre esta modalidad y, por ello, surgen interrogantes que es preciso responder, tales como: qué es la educación a distancia, cuál es su historia, en qué se diferencia de la educación presencial, cuáles son sus características, ventajas y limitaciones, a quién está dirigida y, finalmente, cuál es su nivel de reconocimiento y aceptación en la sociedad actual. Estos interrogantes motivaron la investigación cuyos resultados se presentan en este documento.

Palabras clave: educación, enseñanza y formación, métodos de enseñanza, educación a distancia.

\footnotetext{
${ }^{1}$ Artículo de revisión.

${ }^{2}$ Ingeniero de sistemas; Especialista en docencia universitaria; Magíster en Educación; Doctorando en Ciencias de la Educación. anivarchaves@yahoo.com.
} 


\title{
Distance education, responding to the educational needs of the twenty-first century
}

\begin{abstract}
The information and communications chiefly within technological development offers useful tools to society in different fields, including the education. Moreover, the knowledge society faces new challenges to education systems, and to the people who must follow training processes during all their lives. In this context, the distance education is a critical viable response to a widespread education demand, particularly by people who cannot access classroom education. However, such a mode is little known and therefore there are new urgent questions to answer, such as: What is distance education? What is its history? How differs from the classroom education? What are its features, advantages and disadvantages? Who is targeted to? And what is its level of recognition and acceptance in today's society? These questions resulted in this research whose results are included herein.
\end{abstract}

Keywords: education, teaching and training, teaching methods, distance education.

\author{
A educação a distância como resposta \\ às necessidades educativas do século XXI
}

\section{Resumo}

O desenvolvimento tecnológico, especialmente na área da informação e da comunicação, oferece à sociedade ferramentas úteis nas diferentes esferas, entre essas a esfera da educação; de outro lado, a sociedade do conhecimento proporciona novos reptos aos sistemas educativos e às pessoas que devem continuar um processo de formação vitalício. Neste contexto, a educação a distância erige-se como uma resposta decisiva viável para a demanda generalizada de educação, em particular por pessoas que não podem aceder à formação presencial. No entanto, conhece-se pouco sobre essa modalidade e, por isso, surgem interrogantes que é preciso responder, tais como: o que é a educação a distância, qual a sua história, qual a sua diferencia com a educação presencial, quais são suas caraterísticas, bondades e limitações, a quem está dirigida e, finalmente, qual é seu nível de reconhecimento e aceitação na sociedade atual. Estes interrogantes motivaram a pesquisa cujos resultados são apresentados neste documento.

Palavras chave: educação ensino e formação, métodos de ensino, educação a distância. 


\section{Introducción}

Entre los muchos retos $\mathrm{y}$ necesidades que enfrenta la sociedad actual, un lugar preponderante le corresponde a la educación, en la medida en que la demanda de este servicio en todos los niveles es superior a la capacidad de los sistemas educativos para satisfacerla. En relación con la educación superior, cada vez es mayor el número de personas que aspiran a un cupo en un programa de formación profesional, pues los procesos de selección se tornan más rigurosos y muchos son los aspirantes que no logran ingresar. Adicionalmente, hay un amplio sector que por diferentes motivos, ya sean familiares, laborales, económicos o por el lugar de residencia no pueden aspirar a la educación superior.

En el contexto social, político, económico y cultural del siglo XXI, donde se dan cita la sociedad del conocimiento, la globalización, las TIC y la multiculturalidad, los sistemas de educación convencional son incapaces de atender la demanda de este servicio, mucho más si se piensa en educación a través de toda la vida. En este sentido la educación enfrenta grandes retos en lo referente a cobertura, calidad, incorporación de TIC, actualización de enfoques pedagógicos y curriculares que exigen un análisis de los paradigmas con los que se ha venido trabajando y de las relaciones de la educación con otras áreas de la ciencia y la tecnología.

Frente a estos desafíos, la educación a distancia se erige como una alternativa viable, flexible y eficaz, capaz de asumir un lugar importante en la educación de la sociedad, especialmente si se tiene en cuenta que este modelo recurre a métodos, técnicas y recursos que elevan la productividad y la flexibilidad del proceso de enseñanza y de aprendizaje, ya que no se propone entregar al estudiante un conocimiento terminado, sino desarrollar las habilidades y proporcionar los métodos $\mathrm{y}$ estrategias que le permitan al estudiante continuar su proceso de aprendizaje, apropiar y construir conocimientos, según los necesite, a la vez que permite superar los obstáculos relacionados con el tiempo y el espacio
(Torres, 2004, p. 32; Rodríguez Ruiz, 2013), Alfonso(2003, p. 2) y Contreras, Leal y Salazar $(2001$, p. 7))

La educación a distancia ha tenido un desarrollo prominente en las dos últimas décadas del siglo XX (Begoña, 2004) y al confluir la necesidad del aprendizaje continuo y el desarrollo de las tecnologías de la comunicación, este modelo de educación ha sido puesto a la vanguardia de la práctica educativa (Garrison, 2000 y Rivas, 2012). Para corroborar esta afirmación, Salazar y Melo (2013, p. 86) mencionan que en el mundo existen 21 megauniversidades con programas a distancia que atienden una población aproximada de 12 millones de estudiantes. Estas 21 instituciones están distribuidas así: 14 en Asia, tres en Europa, tres en Norte América y una en África.

Ahora bien, partiendo del papel relevante de la educación a distancia en la sociedad actual, es preciso preguntarse cuánto se conoce acerca de este modelo educativo. Preguntas como qué es la educación a distancia, cuál es su historia, en qué se diferencia de la educación presencial, cuáles son sus características, ventajas y limitaciones, a quien está dirigida y cuál es su nivel de reconocimiento y aceptación en la sociedad actual, conducen a la búsqueda de una mejor perspectiva sobre el tema. En este documento se procura dar respuesta a estas preguntas a partir de los planteamientos de varios expertos en el tema.

\section{Aproximación a la educación a distancia}

No es fácil dar una definición precisa sobre educación a distancia ya que esta modalidad ha cambiado según el tiempo y el lugar donde se desarrolla, pues la que se ofrece hoy es diferente de la que comenzó a impartirse en el siglo XVIII y la de hoy seguramente cambiará en los próximos años; de igual manera como se concibe de forma distinta en los diferentes contextos geográficos (García Aretio, 2002, p. 7) y diferentes autores han propuesto perspectivas diferentes, aunque con algunos elementos en común. Las propuestas al respecto varían en función de factores como: la concepción teórica y filosófica de 
educación, el apoyo político y social, las necesidades educativas de la población, el grupo destinatario, los recursos tecnológicos disponibles y el desarrollo de los medios de comunicación, entre otros. En este trabajo se analizan algunas de las más difundidas, como las de García Aretio (2002), Torres (2004), Moore, Dickson-Deane y Galyen (2011), Contreras, Leal y Salazar (2001), Burns (2011) y Sarmiento (2014).

Para García Aretio (2002, p. 26), la educación a distancia "es un sistema tecnológico de comunicación bidireccional (multidireccional), que puede ser masivo, basado en la acción sistemática y conjunta de recursos didácticos y el apoyo de una organización y tutoría que, separados físicamente de los estudiantes, propician en éstos un aprendizaje independiente (cooperativo)". En esta misma línea, Aliste (2006, p. 16) propone que la educación a distancia es "la combinación de educación y tecnologías de la comunicación para llegar a una audiencia interesada en aprender que está separada por grandes distancias", a la vez que para Torres (2004, p. 37) es "un conjunto de estrategias pedagógicas y mecanismos de comunicación que vinculan a los docentestutores con los estudiantes para desarrollar actividades de enseñanza y de aprendizaje, no coincidiendo en tiempo y espacio geográfico, desarrollándose, por lo general, fuera de los campus universitarios". Mientras que Burns (2011, p. 9) la define como una experiencia de aprendizaje planeada caracterizada por la separación entre el estudiante y el docente, cuya separación es compensada por el intercambio de información y comunicación a través de medios impresos y electrónicos.

En este mismo sentido, para Contreras, Leal y Salazar (2001: 123), el concepto educación a distancia denota formas de aprendizaje que no son guiadas por un profesor en un aula de clases, sino que son planificadas por la institución educativa y se sustentan en materiales didácticos y atención tutorial a través de diferentes medios que facilitan al estudiante acceder a la información y al conocimiento. A su vez, Moore, Dickson-
Deane y Galyen (2011) consideran que la educación a distancia implica un tipo de instrucción en el cual profesor y estudiante se ubican en diferentes momentos y lugares y utilizan diferentes formas de comunicación y de materiales de instrucción. Mientras que Salazar y Melo (2013, p. 102) destacan las relaciones pedagógicas entre estudiantes, docentes e institución, que se establecen mediante el uso de tecnologías y permiten el desarrollo sistémico de procesos formativos de calidad; de forma similar, Cabral (2011, p. 12) concluye que se trata de una modalidad para "impartir conocimientos, habilidades y actitudes mediante actividades seleccionadas, planeadas e institucionalizadas que se encuentran en los materiales de aprendizaje", proceso que requiere una previa definición de las responsabilidades del tutor y del estudiante, mecanismos de seguimiento y evaluación, de igual manera que estrategias y medios de comunicación.

El Ministerio de Educación Nacional de Colombia a través de su Decreto 1295 del 20 de abril de 2010, en su artículo 16 define los programas a distancia como "aquellos cuya metodología educativa se caracteriza por utilizar estrategias de enseñanza-aprendizaje que permiten superar las limitaciones de espacio y tiempo entre los actores del proceso educativo" (p. 10), y en el Decreto 1820 del 1983 se define como el conjunto de actividades y programas educativos donde la relación estudiante-profesor es mediatizada y fomenta que el estudiante aprenda por símismo mediante hábitos de estudio, constancia y autodisciplina. De manera que esta metodología permite ampliar las posibilidades educativas para un importante sector de la sociedad; tal como afirma Jiminián $(2009$, p. 71) se trata de una opción educativa para incorporar al sistema educativo diferentes segmentos de la población que tienen dificultades para cumplir con los requisitos de asistencia que exige la educación presencial. No obstante, el planteamiento más amplio se encuentra en Sarmiento (2014, p. 311) quien considera la educación a distancia como una modalidad incluyente que no aplica restricciones y privilegios de edad, sexo, 
posición social, prerrequisitos ni exámenes de admisión, a la vez que valora la experiencia y conocimientos previos del estudiante y amplía el espacio de aprendizaje, pues no se limita al campus universitario, sino que se extiende al campus de la vida con todas sus dimensiones. En los conceptos de educación a distancia aportados por los autores citados se encuentran elementos comunes como la separación entre el estudiante y el docente, separación que se refiere no sólo al espacio geográfico sino también al momento en que se realizan las actividades de tutoría y de aprendizaje. De igual manera, coinciden en mencionar el uso de recursos tecnológicos o medios de comunicación que hacen posible la interacción entre el estudiante, el docente y los contenidos; sin embargo, difieren en el elemento que ubican como centro de interés, pues algunos dan relevancia a los medios tecnológicos mientras que otros se interesan más por las cuestiones pedagógicas, otros por el aprendizaje y otros por la interacción entre estos elementos. Es precisamente la estrecha articulación con las tecnologías de la comunicación lo que ha permitido un avance significativo en las últimas décadas; no obstante, como forma de educación es ante todo un acto pedagógico y no puede limitarse al uso de medios, también requiere investigación desde la pedagogía y la didáctica.

En el marco de la educación a lo largo de toda la vida, tal como afirma Jacques Delors (1996), y lo corrobora Torres (2004), la demanda de educación por parte de los adultos aumenta significativamente y tiene múltiples formas, con el factor común que este sector de la población no puede acudir regularmente a las aulas de clase por los compromisos y tareas propias de su rol. En este contexto, la educación a distancia se impone como un modelo alternativo y complementario, siendo sus características fundamentales el ser abierta y flexible, las que la hacen idónea para atender esta necesidad social (García Aretio, 2001; Alfonso, 2003).

Desde esta perspectiva, la educación a distancia tiene un carácter innovador por su método y por su flexibilidad, lo que le permite responder a las demandas más disímiles en educación superior. Esto es posible por el uso intensivo de los medios de comunicación y las tecnologías de la información, lo que permite reducir algunos costos y superar la limitación de espacio y tiempo. Por ello, esta modalidad de educación está llamada a "satisfacer el derecho que toda persona tiene a acceder a la enseñanza, en cualquier etapa de su vida y de acuerdo a sus necesidades o intereses". (García Aretio, 2001, p. 2).

Coherente con lo anterior, para la UNESCO la educación a distancia es una oportunidad para que cualquier persona pueda acceder a la educación, y para ello propone que los recursos sean compartidos a través de internet desde la perspectiva cooperativa y colaborativa. Para lo cual es necesario que los Estados formulen políticas basadas en el derecho a la educación, que fomenten el conocimiento, desarrollo y uso de entornos de aprendizaje basados en tecnologías de la información y la comunicación (ACESAD, 2013: 15).

Para el caso de Colombia, a partir del Decreto 2412 (1982), la educación a distancia tiene como objetivos:

- promover cambios mediante la educación para lograr una sociedad más justa,

- fomentar la igualdad de oportunidades de acceso a la educación superior,

- ofrecer oportunidades de educación tecnológica y superior a todos los bachilleres, y

- estimular la creatividad y la investigación.

\section{Abierta y a distancia: dos atributos, un modelo}

Actualmente, es común referirse a la educación a distancia con la denominación de "abierta y a distancia", como si se tratara de un adjetivo compuesto, pero se trata de dos tipos de educación que pueden encontrarse juntas. Bates (1999), García Aretio (2002) y Torres (2004) defienden la necesidad de hacer una distinción entre educación a distancia y educación abierta. En el primer caso, la educación a distancia contrasta con la 
educación presencial en el sentido de que no hay interacción entre el docente y el estudiante en la proximidad de un aula, sino que deben hacerlo a través de los diferentes medios de comunicación; mientras que la educación abierta se refiere a la ausencia de restricciones para el ingreso, pues significa que el servicio educativo se ofrece a todas las personas que deseen tomarlo, donde el estudiante selecciona los cursos y los ritmos de aprendizaje; es la respuesta al derecho de todas las personas a la educación, bajo los principio de democracia, igualdad de oportunidades y justicia social (Contreras, Leal y Salazar, 2001; Cabral, 2011, p. 12). Para García Aretio (2002: 9), la diferencia radica en que la educación a distancia es un modo de entrega de la enseñanza, mientras que la educación abierta sugiere cambios estructurales en el modelo de educación superior que se ha caracterizado por ser selectivo; por su parte, Bates (1999, p.31) considera la educación abierta como una meta o una política de la educación; mientras que la educación a distancia se refiere al medio que hace posible la educación abierta.

En este sentido, Tichapondwa y Tau (2009) consideran que los programas que no tienen límites de edad para el estudiante, no exigen determinado puntaje para el ingreso; el estudiante decide su espacio y tiempo de estudio, cumplen con las dos categorías: abiertos y a distancia. Según estos autores, la diferenciación de educación abierta y educación a distancia sólo es aplicable para los programas a distancia que se estructuran con criterios de educación presencial $\mathrm{y}$, por tanto, no son abiertos.

En este punto del debate es importante presentar el planteamiento de González (2003), quien reconoce que educación abierta y educación a distancia no significan lo mismo, pues la primera se sustenta en la flexibilidad tanto en lo concerniente al ingreso al sistema educativo como al desarrollo del proceso de aprendizaje, mientras que la segunda se refiere a lautilización de mediaciones para superar las limitantes de espacio y tiempo, dicho en otros términos, a la separación entre el estudiante y el profesor. No obstante considera que es importante apostarle a la consolidación de la educación abierta y a distancia como un nuevo modelo educativo basado en principios de flexibilidad, pertinencia y autonomía. Redefinir el concepto de educación abierta y a distancia, ya no como dualidad sino como unidad, implica: fortalecer la cultura educativa en torno a este modelo, diseñar estrategias pedagógicas y didácticas que hagan posible la autogestión del conocimiento, construir escenarios educativos inspirados en la autoformación y consolidar comunidades académicas que se definan no por la presencialidad, sino por procesos de aprendizaje cooperativo y colaborativo.

\section{Génesis de la educación a distancia}

Con el ánimo de establecer un momento de partida para lo que hoy se conoce como educación a distancia, Alfonso (2003) y Arboleda (2013) se remontan a civilizaciones antiguas como la sumeria, egipcia, hebrea y griega, y proponen que la primera forma de educacióna distancia se realizómediantecartas, práctica que se conoce como epistolografía, mientras que Iriondo y Gallego (2013) al igual que Simpson y Anderson (2012) sugieren que este tipo de educación existe desde que se inventó la imprenta, ya que antes de este evento la enseñanza era exclusivamente oral, fue mediante el texto impreso que se pudo distribuir el conocimiento a distancia; luego, con el surgimiento del sistema postal, se organiza el modelo de educación por entregas. Sin embargo, la educación a distancia que hoy se conoce pudo haber comenzado a desarrollarse en el siglo XVIII o el XIX, pero no es fácil precisar su inicio por cuanto no hay consenso entre los investigadores. Por una parte, Alfonso (2003) y Bower y Hardy (2004) sostienen que el primer curso se ofertó en 1728 , cuando en la Gaceta de Boston el profesor Caleb Philips anunció la apertura de un curso de taquigrafía con material autoinstructivo con entregas semanales y tutoría. Por otra parte, Gunawardena y McIsaac (2004) afirman que el primer programa de educación a distancia lo ofreció la Universidad de Chicago a finales de 1800, pensando en las 
personas que no disponían del tiempo para residir en una institución educativa. Un tercer planteamiento ubica el inicio de la educación a distancia en 1833 cuando la Universidad de Swedish anuncia la oportunidad de estudiar composición por correspondencia y aproximadamente sobre 1840 Isaac Pitman adaptó el sistema de taquigrafía para impartir formación bíblica por correspondencia, lo que conllevó a la creación de Sir Isaac Pitman Correspondence Colleges (Bower y Hardy, 2004, p. 6). De otro lado, Contreras, Leal y Salazar (2001) sugieren que comenzó en la Universidad de Londres alrededor de 1850 y que su interés no era la enseñanza sino la organización de exámenes.

En cualesquiera de los casos, los autores coinciden en que la educación a distancia tomó fuerza, se expandió y ganó reconocimiento en la segunda mitad del siglo XX, aunque se proponen diferentes décadas; mientras que García Aretio (2001) sostiene que fue a partir de la década de 1960, Moreno (2010) propone que fue a partir de 1970, mientras que Garrison (2000) sugiere que ocurrió en las dos últimas décadas del siglo XX.

También es importante resaltar que desde que surgió este tipo de educación ha recibido diferentes denominaciones atendiendo a diferentes criterios, como sus fundamentos, características, acciones e impacto (ACESAD, 2013). Entre las denominaciones que ha recibido, García Aretio (2001) menciona: educación por correspondencia, estudio independiente, forma industrializada de educación, estudios externos, aprendizaje flexible y autoformación. Según Gunawardena y McIsaac (2004), en Estados Unidos, surgió como educación por correspondencia, pero a partir de 1982 el concepto más amplio es educación a distancia, dentro de éste tienen cabida otros conceptos como: aprendizaje abierto, aprendizaje en red, aprendizaje flexible, aprendizaje distribuido y aprendizaje en el ciberespacio. También Tichapondwa y Tau (2009) y Moore, Dickson-Deane y Galyen (2011) coinciden en que el concepto de educación a distancia es el más general y comprende otras denominaciones que tiene como elemento común el hecho que el estudiante no hace presencia física en una institución, sino que realiza sus actividades académicas desde un lugar distinto, como puede ser su casa. Desde que se incorporó el uso del computador y la red internet en los procesos educativos a distancia, se ha utilizado indistintamente denominaciones como e-learning, educación en línea y educación virtual. Para algunos autores, como se verá más adelante, estas denominaciones se refieren a la cuarta y quinta generación de la educación a distancia.

\section{La evolución de la educación a distancia}

La evolución de la educación a distancia se ha dividido en generaciones o etapas teniendo en cuenta principalmente la tecnología utilizada para producir y distribuir los recursos educativos, así se encuentran autores que proponen tres momentos, otros cuatro, cinco y hasta seis. Ramírez (2012), CNA (2012) y Arboleda (2013), por ejemplo, relacionan tres generaciones. La primera se caracterizó por el uso de documentos impresos que eran distribuidos mediante el servicio de correo; en la segunda se continuó utilizando los módulos y el correo, pero se incorporó la radio y la televisión en menor o mayor proporción; la tercera está marcada por la utilización de medios electrónicos, con un papel preponderante de las redes telemáticas. Simpson y Anderson (2012) y Aparici (2002), por su parte, hacen referencia a cuatro generaciones; la primera se caracterizó por el uso de material impreso y del sistema de correspondencia, por el intercambio de libros, manuales y otros documentos; desde la perspectiva social, esta generación estuvo motivada por la búsqueda de la justicia social, principalmente, ofreciendo educación a las mujeres y la clase trabajadora que no podía acudir a las instituciones educativas. La segunda generación se reconoce por la utilización de tecnología analógica como la radio, la televisión y los videos, cuyo uso de esta tecnología permitió una ampliación significativa de la cobertura. La 
tercera incluye la informática y el uso de documentos electrónicos con prevalencia de la comunicación en un sentido y de forma asíncrona. La cuarta se podría llamar digital e integra diversos medios tecnológicos a través de redes, principalmente internet y sus servicios, permitiendo comunicación en ambos sentidos y actividades sincrónicas como teleconferencias. Ahora bien, el inicio y fin de las etapas son difusos, pues en cada una se encuentra una mezcla con tecnologías de las etapas anteriores.

La Asociación Colombiana de Educación Superior a Distancia-ACESAD (2013) propone cinco generaciones que comienzan con la educación por correspondencia y llega hasta la educación a través de entornos virtuales. Las cuatro primeras coinciden con las mencionadas por Aparici (2002), y aumenta la quinta generación que incluye mayor integración y acceso a contenidos mediante repositorios y campus virtuales. Leal (2013) considera que la más reciente generación de la educación a distancia es la virtual, la cual se basa en la interacción y la interactividad entre estudiantes, medios, mediaciones, mediadores, actividades, evaluación con calidad, desde la premisa del aprendizaje autónomo, complejo, significativo y colaborativo. En Colombia, el Ministerio de Educación Nacional MEN reconoce la modalidad virtual y, a través del Decreto 1295 de 2010, establece las condiciones mínimas de calidad para programas ofrecidos bajo esta modalidad.

Gunawardena y McIsaac (2004) tienen en cuenta la utilización de seis avances tecnológicos que caracterizaron la educación a distancia, así: en la primera predominan los medios impresos y el sistema de correo, en la segunda la difusión por radio y televisión, la tercera incluye la televisión por cable, la cuarta aplica televisión interactiva, la quinta archivos de audio y video, y la sexta utiliza el sistema de teleconferencia.

Hasta el momento se han presentado planteamientos que se enfocan en la tecnología utilizada para la difusión o entrega de los contenidos; no obstante, hay otras miradas sobre cómo ha cambiado la educación a distancia a través de los años. En este sentido se puede mencionar a Anderson y Dron (2011) quienes reconocen la importancia de los desarrollos tecnológicos y cómo éstos afectan y determinan las formas de ver el mundo y, por ende, los procesos educativos, pero no están de acuerdo en que la educación a distancia se clasifique tomando como parámetro la tecnología utilizada. Ellos proponen tres generaciones con base en los enfoques pedagógicos aplicados. Así, la primera generación se basa en el cognitivismoconductista; la segunda en el constructivismo social; y la tercera en el conectivismo. Garrison (2000: 2) considera que en el siglo $\mathrm{XX}$ el interés de la educación a distancia se enfocó en "superar las barreras de tipo geográficas mediante estrategias organizacionales como la producción en masa y la entrega de paquetes de aprendizaje", lo que le ganó el nombre de educación industrializada. En el siglo XXI, el centro de interés se ha movido hacia la enseñanza y el aprendizaje y hacia las tecnologías y estrategias de comunicación que permiten mantener un apoyo sostenido, en cualquier momento y en cualquier lugar.

\section{Características de la educación a distancia}

Son muchas las características de la educación a distancia que la distinguen de otras formas de educación, según lo han planteado autores como García Aretio (2002), Saba (2003), Florido y Florido (2003), Begoña (2004) y Cabral (2011). Aquí se revisan las más importantes a juicio del autor.

- El protagonista es el estudiante: la característica fundamental de la educación a distancia es que el centro del proceso educativo lo constituyen el estudiante, $\mathrm{su}$ responsabilidad y sus interacciones. El aprendizaje autónomo es el enfoque por excelencia en educación a distancia. La responsabilidad del estudiante sobre su aprendizaje encuentra su expresión plena en la relación con el docente en una conversación didáctica guiada. Esto la diferencia de cualquier otro modelo 
educativo (Saba, 2003). El estudiante no es un receptor de conocimiento, sino que contribuye a la construcción del mismo y a la calidad de la experiencia de aprendizaje (Iriondo y Gallego, 2013).

- La relevancia de los recursos educativos: un lugar privilegiado le corresponde al material educativo en el marco de la educación a distancia y del aprendizaje autónomo, según Begoña (2004), adquiere un valor especial por cuanto contiene toda la información que se ofrece al estudiante, tanto los temas de estudio como la descripción de las actividades de aprendizaje y la estrategia de evaluación. Al no existir la interacción directa entre docentes y estudiantes, se produce un cambio en la forma de llevar a cabo la enseñanza y esa forma es a través del uso de materiales de estudio debidamente planificados, diseñados y elaborados. De esto se deduce un compromiso muy grande para las instituciones y los docentes que desarrollan los materiales de estudio, ya que éstos requieren diseño instruccional, estructura y organización, de tal manera que faciliten el aprendizaje al estudiante (Sarmiento (2014, p. 312).

- El uso de recursos tecnológicos: la educación en general y la educación a distancia en particular se han nutrido y fortalecido con el avance de los recursos técnicos de comunicación de masas; desde el papel hasta las herramientas telemáticas, pasando por el correo, el teléfono, la radio y la televisión, se han utilizado en la educación a distancia. El uso de los medios para hacer asequible el conocimiento a los estudiantes es determinante en el diseño de los cursos a distancia y suele ser la base de la cobertura y la economía de los programas educativos (Bates, 1999). Para García Aretio (2002: 24) “Toda acción educativa eficaz acaba convirtiéndose en una técnica apoyada en una ciencia". En educación a distancia, más que en la presencial, la planificación sistemática y rigurosa, tanto en lo institucional como en lo pedagógico, resulta imprescindible. La falta de planeación del componente tecnológico en educación a distancia puede conducir a errores en el diseño, producción y distribución de los materiales de estudio, fallas en la coordinación de la interacción entre los distintos recursos personales y materiales, de igual manera que a incoherencias en la evaluación de los aprendizajes en función de los propósitos.

La autonomía: el estudiante puede escoger con autonomía el espacio, el tiempo, el estilo, el ritmo y el método de aprendizaje, con base en sus propias capacidades y posibilidades (García Aretio, 2002). El estudiante tiene la comodidad de aprender desde cualquier lugar y también de gestionar su tiempo, y esto le da la posibilidad de complementar la educación con cualquier otra actividad, ya sea doméstica o profesional (Florido y Florido, 2003). No obstante, la gestión del tiempo por parte del estudiante se constituye en una variable crítica para el éxito en la educación a distancia, debido a que el estudio y el trabajo pueden entrar en conflicto cuando no hay tiempo suficiente para las dos dimensiones o cuando la falta de organización lleva a que las actividades se mezclen.

- Autorregulación: si por un lado se tiene la autonomía, por el otro está la autorregulación, entendida como la acción reguladora que una persona ejerce sobre los distintos momentos y actividades relacionados con su propio proceso de aprendizaje. Para García Martín (2012, p. 206), el aprendizaje responde a una actividad intencional y consciente que busca una conducta significativa y estratégica; por tanto, el aprendizaje debe ser reflexivo, autónomo y autodirigido; esto es importante de manera especial en educación a distancia, donde la responsabilidad sobre los resultados del proceso educativo recaen directamente sobre el estudiante y no sobre el profesor, como en otros modelos educativos. Adicionalmente, la educación a distancia exige competencias por parte del estudiante, especialmente: competencias 
de lectura comprensiva, identificación y solución de problemas, investigación, análisis y comunicación (Florido y Florido, 2003, p. 5).

- La separación estudiante-docente: los programas de educación a distancia están diseñados para manejar determinado grado de separación física y temporal entre el estudiante y el profesor; por tanto, el proceso de aprendizaje no tiene supervisión inmediata del profesor, la comunicación es mediada y el contacto cara a cara no es frecuente, como sí ocurre en el modelo de educación presencial. La separación física y temporal entre el estudiante y el profesor no es completa, debido a que los cursos a distancia pueden incluir encuentros presenciales y sesiones de comunicación síncrona por vía telefónica o mediante el uso de herramientas de Internet. (García Aretio, 2002).

- El apoyoy tutoría: la forma convencional de educación consiste en la inscripción a una institución educativa donde el aprendizaje se lleva a cabo mediante el contacto cara a cara con el docente y el grupo; otra forma de educación es la autodidacta, donde el estudiante aprende por sus propios medios sin contar con la guía y supervisión de ninguna institución o profesor. Entre estos dos extremos se ubica la educación a distancia, en la que el aprendizaje se lleva a cabo en cierta medida de forma autodidacta pero contando el estudiante con tutoría por parte de una institución, que se encarga de apoyarlo, motivarlo, facilitarle el aprendizaje y evaluarlo. Como lo refiere Begoña $(2004$, p. 5), el apoyo y la tutoría puede ser muy variado, tanto en su frecuencia como en su duración; puede tratarse de encuentros presenciales en los que el tutor desarrolla los temas o puede no haber encuentros presenciales, en cuyos casos el tutor resuelve las preguntas que el estudiante le hace llegar por diferentes medios. También están las tutorías a través de entornos virtuales de aprendizaje, donde la comunicación se realiza a través de herramientas telemáticas.
- La interacción: para que haya un proceso educativo debe existir comunicación completa, en ambas direcciones y con su correspondiente realimentación, entre el estudiante y el docente. Como afirma Sangrá (2002, p. 2), la interacción es algo positivo en la educación y en las primeras generaciones había sido una de las debilidades del modelo de educación a distancia, pero gracias a las TIC, en la tercera generación es posible una interacción continua. En educación a distancia, el diálogo puede comenzarlo el docente a través de las actividades de aprendizaje o puede comenzarlo el estudiante para aclarar las dudas que le suscite el material de estudio; en algunos casos esta comunicación puede ser multidireccional en cuanto involucra no sólo al estudiante y al docente, sino también a los compañeros. La particularidad de la comunicación en la educación a distancia es que no se lleva a cabo de forma directa, sino haciendo uso de diversos medios de comunicación. En este sentido Lozano y Tamez (2014, p. 197) refieren que una realimentación de calidad debe mostrarle al estudiante hacia dónde va, cuál es el objetivo o meta de las actividades y los aprendizajes, cómo es su desempeño, qué seguirá en el proceso y cómo se relaciona lo que está haciendo con las actividades que siguen.

- La cobertura: el uso de medios de comunicación hace posible que se eliminen las barreras espacio-temporales y la educación a distancia llegue a rincones insospechados de la geografía, donde las personas no tienen oportunidad de acceder a la educación superior en otra modalidad (Torres, 2004; García Aretio, 2001; Arboleda, 2013). Así, la educación a distancia está al servicio de un amplio sector de la población que por habitar en regiones alejadas de las instituciones de educación superior o por sus compromisos laborales y familiares no puede vincularse a programas presenciales. Estas características han hecho que el 
número de estudiantes a distancia crezca continuamente al igual que la oferta de programas académicos. En Colombia, según Estévez, Castro y Rodríguez (2014, p. 4), la oferta de programas en modalidad a distancia y virtual es de aproximadamente el $7 \%$ de la oferta de educación superior.

\section{Ventajas de la educación a distancia}

Algunas de las características de la educación a distancia constituyen una ventaja frente a otras formas de educación, dependiendo del contexto en que se desarrolle el proceso educativo y de algunos elementos como la institución que la ofrece, los materiales y recursos utilizados, los estudiantes y sus necesidades.

La asincronicidad en el desarrollo de las actividades de aprendizaje es, sin lugar a dudas, una ventaja importante de este modelo de formación (Facundo Díaz, 2009, p. 147). El estudiante no necesita coincidir temporalmente con el docente para el desarrollo de las interacciones pedagógicas, lo que le permite al primero decidir el día y la hora en que le es más propicio dedicarse a sus actividades académicas. El estudiante dispone de más tiempo para reflexionar antes de dar respuesta a una cuestión, puede hacer uso de recursos sin interrumpir el flujo de la discusión o del estudio de un tema; además, cuenta con registros de las interacciones como correos, foros, boletines, que pueden ser utilizados para la investigación o la evaluación (King, et al., 2001).

La autonomía del estudiante es otra ventana importante. En educación a distancia, el estudiante es autónomo para decidir en qué lugar estudia, en qué momento y a qué ritmo, sin depender del profesor o de los compañeros. Esto permite que personas con diferentes ocupaciones y estilos de vida puedan acceder a programas de estudio, sin separarse de su entorno laboral o familiar. En algunos casos, el lugar de residencia o el entorno de trabajo pueden constituirse en facilitadores del proceso de aprendizaje en la medida en que permiten la experimentación o la confrontación de los contenidos. Ante la ausencia del profesor, el estudiante asume el control de su proceso de aprendizaje y se convierte en el protagonista del mismo, de esta manera se erige como sujeto activo, motivado y comprometido que se esfuerza por aprovechar el tiempo y los recursos disponibles para conseguir sus metas, lo que le reportará un aprendizaje adicional que se verá reflejado en sus actuaciones futuras. También se destaca la mayor cobertura de la que es capaz la educación a distancia, ya que no existe la limitante de la cantidad y el tamaño de las aulas; los estudiantes pueden estar en diferentes lugares y no tienen que reunirse en un mismo espacio, excepto en casos extraordinarios (García Aretio, 2001 y Arboleda, 2013). Esto es muy importante dado que muchos países enfrentan el problema de falta de cobertura del servicio educativo. Al respecto, Torres (2004: 37) afirma que la educación a distancia "constituye una alternativa importante para ampliar la cobertura, propiciando que sectores mayores de población realicen estudios universitarios y que los profesionales de distintos campos del saber puedan llevar a cabo procesos de actualización". Como ejemplos de la gran cobertura de que hace gala la educación a distancia se tiene la Indira Gandhi National Open University de la India, con aproximadamente tres millones de estudiantes; la Allama Iqbal Open University de Pakistán, con un millón ochocientos mil estudiantes y la Anadolu University de Turquía, con cerca de ochocientos ochenta mil estudiantes (Salazar y Melo, 2013, p. 86) y en Colombia, la Universidad Nacional Abierta y a Distancia UNAD, ha superado los 70 mil estudiantes en algunos periodos académicos.

Desde la perspectiva económica se observa que se presenta una disminución en los costos educativos. Aunque inicialmente la producción de los materiales y la puesta en marcha de un proceso de educación a distancia puede tener un costo alto para la institución, esto se verá compensado por la cantidad de estudiantes que pueden hacer uso de los mismos; mientras que para los estudiantes se disminuyen los costos asociados al transporte, alojamiento 
y alimentación que serían necesarios en educación presencial (García Aretio, 2001; Arboleda, 2013).

También constituye una ventaja, desde la perspectiva de Tichapondwa y Tau (2009), el enfoque didáctico con que se diseñan los materiales, lo que hace que el estudiante aprenda sin necesidad de tener al docente en frente; el estudio es dirigido, pero el estudiante cuenta con autonomía para acomodarlo a sus circunstancias y la flexibilidad en el manejo del tiempo de estudio.

Otra ventaja no menos relevante que las anteriores consiste en que los adultos no necesitan renunciar a su trabajo para poder adelantar sus estudios, ya que esta modalidad de educación permite ejercer las dos actividades y como se puede constatar en el estudio realizado por Facundo Díaz (2009, p. 125), aproximadamente las tres cuartas partes de la población estudiantil a distancia son personas que trabajan.

\section{Limitaciones de la educación a distancia}

Tal como se ha mostrado, la educación a distancia ofrece grandes posibilidades y también presenta algunos inconvenientes, como los que se esbozan a continuación. Por una parte, la educación es un acto de socialización y esto aplica también para la educación a distancia, pero en muchos casos se limita a las interacciones pedagógicas. El mayor inconveniente es que el estudiante se encuentra aislado ya que la interacción con el tutor y los compañeros suele ser escasa, además es frecuente que el estudiante tenga dificultades para acceder a los recursos educativos (Tichapondwa y Tau, 2009). El estudiante tiene pocas oportunidades de socializar con los docentes y con los compañeros, así como pocas ocasiones para participar en actividades culturales, deportivas, comunitarias o religiosas promovidas por su institución. El sentimiento de soledad que experimentan los estudiantes de educación a distancia es característico de esta modalidad (Correa, 2012, p. 72), pero en la medida que la educación a distancia evoluciona enfoca sus esfuerzos a disminuir o eliminar dicho sentimiento de soledad, y para ello se llevan a cabo los encuentros tutoriales y actualmente se utilizan diferentes herramientas telemáticas para ofrecer acompañamiento a los estudiantes. Por otra parte, en el proceso educativo el estudiante aprende en función de los contenidos estudiados y la experimentación en el laboratorio, pero también se producen cambios de actitud en función del contacto directo con el profesor y con los compañeros. En la educación a distancia, al reducirse las oportunidades de encuentro presencial también disminuye la posibilidad de movilizar actitudes por el trato directo con las demás personas (García Aretio, 2001; Florido y Florido, 2003).

Otro aspecto importante en el proceso educativo es la realimentación que el docente ofrece a los estudiantes en relación con su desempeño en las diferentes actividades pedagógicas, y ésta es mucho más provechosa cuanto más cercana sea al momento en que ocurre el acierto o el error; en la interacción directa entre profesor y estudiantes la realimentación puede ser inmediata, mientras que en educación a distancia es más lenta y en algunos casos puede llegar cuando el estudiante ya ha avanzado a otra temática o actividad. Aunque muchas herramientas telemáticas posibilitan una comunicación sincrónica, esto no siempre es posible debido a las diferencias en el manejo del tiempo y a la cantidad de estudiantes asignados a cada tutor. Los estudiantes que ingresan a programas de educación a distancia provienen de modelos de educación presencial, por ello experimentan dificultades y tardan un tiempo en adaptarse al modelo de educación a distancia. Este modelo requiere que el estudiante posea competencias para la lectura e interpretación, de igual manera que para la escritura (Florido y Florido, 2003, p. 5), ya que la comunicación con el docente y los compañeros se realiza principalmente de forma escrita, pero los estudiantes no siempre ingresan con estas competencias, lo que constituye un inconveniente tanto para el estudiante como para los docentes. Según Contreras, Leal y Salazar (2001, p. 142), los 
estudiantes que ingresan a la modalidad a distancia enfrentan dificultades para realizar lectura crítica y comprensiva, para comunicar sus ideas y propuestas y para organizar de forma eficiente sus actividades de estudio en el tiempo disponible.

Algunos estudiantes tardan mucho o no logran desarrollar la autodisciplina y pierden cursos, incluso pueden llegar a abandonar sus estudios (García Aretio, 2001). Por otra parte, al tratarse de educación abierta los estudiantes ingresan con un mínimo de requisitos, eso hace que muchos ingresen después de haber sido rechazados en otras instituciones por no contar con los altos niveles de competencia y habilidad de pensamiento que éstas exigen, lo que hace que en el imaginario cultural se lea la educación abierta y a distancia como facilista (González, 2003).

Hay quienes dudan de la capacidad de la educación a distancia para lograr los propósitos educativos y esta duda puede ser razonable si las instituciones no garantizan que los materiales de estudio son desarrollados por profesionales altamente cualificados y si el proceso educativo no se planifica adecuadamente (García Aretio, 2001). De igual manera, la calidad del proceso educativo se ve amenazada cuando las instituciones incorporan docentes sin formación $\mathrm{o}$ experiencia en educación abierta y a distancia por lo que intentan desempeñarse como en la educación tradicional. También se presenta el caso de instituciones que ofrecen bajos salarios, lo que conlleva a falta de compromiso por parte de los docentes (González, 2003). Los estudiantes son heterogéneos, pero los materiales de estudio y los métodos son los mismos para todos, esto unido a la falta de interacción directa entre estudiante y profesor puede significar una dificultad para algunos estudiantes (García Aretio, 2001). También ocurre que las instituciones producen grandes cantidades de materiales los cuales resultan muy difícil o costoso de actualizar, por lo que se mantienen durante un tiempo prolongado $\mathrm{y}$, por tanto, cuando llegan al estudiante son obsoletos (González, 2003).

Otro aspecto de gran importancia es el proceso de evaluación del aprendizaje, el mismo que representa un reto para las instituciones y los docentes, principalmente en lo concerniente a la autenticidad, debido a que el estudiante tiene mayores oportunidades de cometer plagio o hacer fraude. En algunos casos es muy difícil establecer la autoría del trabajo o la autenticidad de quien presenta una prueba (García Aretio, 2001).

Por otra parte, Aparici (2002) se refiere a la importancia de la participación o resistencia de los estudiantes en los sistemas educativos y considera que en la educación a distancia, en mayor grado que en la tradicional, los centros de enseñanza o los docentes imponen un totalitarismo, sin que los estudiantes puedan expresar su pensamiento al respecto. Esta posición se corrobora al observar la realidad de muchas instituciones donde los cursos se presentan como producto terminado a los estudiantes, quienes deben limitarse a seguir la programación (agenda de actividades). En este sentido, Garrison (2000) menciona que muchas veces las iniciativas son lideradas por el poder político o por expertos en tecnología y ellos, por lo general, carecen de una comprensión coherente sobre la práctica de la educación a distancia y la gama de posibilidades para alcanzar los resultados deseados. Algunos administradores educativos se enfocan no en las cuestiones educativas, sino en las implicaciones financieras, en disminuir costos y generar beneficios.

\section{Reconocimiento social de la educación a distancia}

La educación a distancia fue una propuesta que revolucionó el campo de la educación ya que rompía el paradigma del aula de clase y de la educación como privilegio de ciertos grupos sociales, y esto hizo que hubiera resistencia por parte de los sectores más conservadores. Gunawardena y McIsaac (2004) mencionan que en el momento que surge la educación por correspondencia en Estados Unidos, la educación superior estaba diseñada sólo para las élites, por eso este modelo de educación fue visto como inferior y muchos educadores 
lo miraban como un simple negocio. En los primeros años fue considerado antidemocrático y una ofensa para dichas élites; sin embargo, ofrecer iguales oportunidades de acceso a la educación a toda la población siempre ha sido parte de los ideales democráticos norteamericanos, lo que permitió que la educación por correspondencia tomara un nuevo rumbo.

Frente a la descalificación de la educación a distancia, es preciso citar a King et al. (2001), quienes reconocen que prevalece el supuesto que la educación a distancia es inferior a la educación presencial, pero defienden la idea contraria, considerando que cada tipo de educación tiene sus propias fortalezas y limitaciones, y si la educación a distancia presenta algunas limitantes al no llevar a cabo las interacciones persona a persona, también tiene fortalezas que el sistema tradicional no posee, como el manejo del tiempo con autonomía del estudiante para llevar su propio ritmo de aprendizaje, la gestión de contenidos, el registro de las interacciones a través de los medios de comunicación, entre otras.

Asimismo, Matallana y Torres (2011) afirman que la educación a distancia adquiere cada vez más relevancia en cuanto cambia los paradigmas educativos y promueve que las instituciones de educación superior se conviertan en centros generadores de conocimiento ya que, además de superar las barreras de tiempo y espacio, acogen a todos los estudiantes y les permiten continuar su formación sin tener que cambiar su vida laboral, social y familiar. En esta perspectiva, Parra (2013) asegura que en la actualidad se reconoce la importancia y la validez de las modalidades a distancia y virtual como posibilidades concretas frente a los retos y limitaciones relacionadas con inclusión, cobertura, calidad y pertinencia de la educación.

En este sentido, Arboleda (2013, p. 48) afirma que "[...] la educación a distancia es reconocida en el ámbito internacional como estrategia legítima para adelantar el proceso de enseñanza-aprendizaje de manera predominantemente no presencial, en cualquier nivel, ampliando la cobertura del servicio educativo sin menoscabo de la calidad". Desde esta misma perspectiva, Barden (2014, p. 810) asegura que la educación a distancia y la educación virtual llegaron para quedarse y pueden ser tan eficaces como la educación presencial. La educación a distancia es la preferida por los estudiantes adultos y cada vez son más las personas que continúan o retoman sus estudios en modalidad a distancia o virtual, especialmente por la flexibilidad que ofrecen estos modelos, lo que les permite adelantar sus estudios y seguir con sus demás actividades. Y como afirma Rodríguez (2014, p. 76), la sociedad del conocimiento exige a las personas un proceso continuo de actualización de conocimientos y capacidades para mejorar su desempeño de manera permanente, lo que ha llevado a que un porcentaje alto de la población adulta acuda a programas de educación a distancia.

\section{Conclusiones}

La educación a distancia es una modalidad que difiere de la educación convencional en varios aspectos, pero dichas diferencias no la hacen mejor ni peor, simplemente diferente. Utiliza diferentes estrategias para llegar a los estudiantes y promover el aprendizaje, diferentes medios, diferente modelo de aprendizaje y está orientada a un sector de la sociedad que la educación presencial no puede atender. Tal como se mostró en la primera parte del documento, la educación a distancia es un sistema que se apoya fuertemente en la tecnología disponible para alcanzar sus propósitos que no son otros que hacer posible el acceso a la educación para todas las personas, independiente del lugar de residencia y de sus ocupaciones. Es un modelo flexible que le permite al estudiante decidir sus estrategias de estudio, sus horarios y su ritmo de aprendizaje, de tal manera que se configuren en torno a sus circunstancias y que no sea el estudiante quien deba acomodarse a las condiciones de las instituciones y programas educativos.

Sobre dónde y cómo comenzó la educación a distancia hay diferentes opiniones, pero ya sea que comenzara en culturas antiguas, en Reino 
Unido o en Norte América, desde sus inicios ha buscado que las mujeres, los trabajadores y personas de los estratos socio-económicos bajos tengan oportunidad de acceder a la educación, ha conseguido ampliar la cobertura y superar las barreras de espacio y tiempo, logrando así cumplir con su propósito social de ofrecer educación a todas las personas con equidad y sin distinción alguna.

Entre las características de la educación a distancia vale la pena destacar algunas que le dan identidad como son el protagonismo del estudiante, la flexibilidad, el uso de recursos tecnológicos, el papel de los recursos educativos y la separación entre estudiantes y docentes. Algunas de estas propiedades pueden resultar ventajosas para los estudiantes $\mathrm{y}$ algunas otras desfavorables, pues mucho de esto depende de las circunstancias y el perfil del estudiante. Si bien para algunos la autonomía significa la oportunidad de tomar sus propias decisiones y de tener el control sobre el proceso educativo, para otros puede significar una dificultad en la medida en que no cuentan con la disciplina y la responsabilidad suficientes para hacerse cargo de su proceso formativo. Aun así, aún hay muchos aspectos que requieren ser evaluados, mejorados y supervisados constantemente, ya sea porque corresponden a problemas no resueltos, como el riesgo de plagio y de suplantación, o porque se refieren a asuntos cambiantes que exigen actualización constante, como son los materiales educativos y las estrategias comunicativas entre docentes y estudiantes.

Para finalizar, la educación a distancia ha sido objeto de críticas, algunas con fundamento y otras sin él; ha enfrentado resistencia desde sus inicios y se ha cuestionado su capacidad para formar profesionales competentes; ha tenido sus defensores y sus detractores; no obstante, se ha mantenido, ha crecido y se ha fortalecido en el curso de aproximadamente doscientos años; en la actualidad, aproximadamente doce millones de personas estudian a distancia y muchos millones han estudiado y cosechan los frutos de dichos procesos de formación; por tanto, frente a las limitaciones y debilidades que este modelo educativo pueda presentar, se requiere investigación, propuestas y apoyo tanto de la sociedad como del gobierno y las instituciones, porque la educación a distancia seguirá avanzando y satisfaciendo la necesidad de educación de un amplio sector de la población.

\section{Referencias}

Alfonso, I. (2003). La educación a distancia. Acimed V. 11 (1). Recuperado de http://scielo.sld.cu/scielo. php? script $=$ sci_is suetoc \& pid $=1024$ $943520030001 \& \operatorname{lng}=\mathrm{es} \& \mathrm{nrm}=$ iso

Aliste Fuentes, C. (2006). Modelo de comunicación para la enseñanza a distancia en internet. (Tesis doctoral). Universidad Autónoma de Barcelona.

Anderson, T. \& Dron, J. (2011). Three Generations of distance education pedagogy. International Review of Research in open and distance learning, 12 (3)

Aparici, R. (2002). Mitos de la educación a distancia y de las nuevas tecnologías. Revista Iberoamericana de Educación a Distancia RIED, 5 (1). Recuperado de http://ried. utpl.edu.ec/sites/default/files/files/pdf/v5-1/ volumen5-1.pdf

Arboleda, N. (2013). La nueva relación entre tecnología, conocimiento y formación tiende a integrar las modalidades educativas. En Arboleda, Néstor y Rama, Claudio. (Ed.), Educación superior a distancia y virtual en Colombia. pp. 47-62. Bogotá: Virtual Educa.

Asociación Colombiana de educación superior a distancia-ACESAD. (2013). Lineamientos conceptuales y criterios de calidad de la modalidad de educación a distancia. Bogotá. Recuperado de http://www.acesad.org.co/ Presentaciones/propuestapoliticapublica.pdf

Bates, T. (1999). La tecnología en la enseñanza abierta y la educación a distancia. México: Trillas. Recuperado de http://www.facmed. 
unam.mx/emc/computo/infoedu/modulos/ modulo2/material2a.pdf

Begoña Tellería, M. (2004). Educación y nuevas tecnologías. Educación a Distancia y Educación Virtual. Revista de Teoría y Didáctica de las Ciencias Sociales, 9, 209222. Recuperado de http://www.redalyc.org/ pdf/652/65200912.pdf

Bower, B. \& Hardy, K. (2004). From correspondence to cyberspace: changes and challenges in distance education. New directions for community colleges, 128, 5-12. Recuperado de http://onlinelibrary.wiley.com/ doi/10.1002/cc.v2004:128/issuetoc

Burns, M. (2011). Distance Education for TeacherTraining: Modes, Models and Methods. Washington: Education Development Center. Recuperado de http://idd.edc.org/sites/idd. edc.org/files/Distance $\% 20$ Education $\% 20$ for $\% 20$ Teacher $\% 20$ Training $\% 20$ by $\% 20$ Mary\%20Burns\%20EDC.pdf

Cabral Vargas, B. (2011). La educación a distancia desde la perspectiva bibliotecológica. Universidad Nacional Autónoma de México. Recuperado de http://132.248.242.3/ publica/ archivos/libros/educacion_distancia_ bibliotecologica.pdf

Consejo Nacional de Acreditación-CNA. (2006). Indicadores para la autoevaluación con fines de acreditación de programas de pregrado en las modalidades a distancia y virtual. Bogotá: Corcas editores.

Contreras, M. E.; Leal, J. y Salazar, R. (2001). Educación a distancia: respuesta a la formación profesional en un mundo globalizado. Bogotá: Ediciones hispanoamericanas.

Correa Granda, C. (2012). Videos temáticos: recursos de apoyo a la tutoría de estudiantes. En Asociación Iberoamericana de Educación Superior a Distancia. (2012). Buenas prácticas en educación a distancia. Universidad Técnica Particular de Loja.
Darden, D. (2014). Relevance of the Knowles Theory in Distance Education. Creative Education, 5, 809-812. Doi: http://dx.doi. org/10.4236/ce.2014.510094

Delors, J. (1996). La educación encierra un tesoro. Madrid: Santillana.

Enríquez Vázquez, L. (2012). Nuevos modelos de educación abierta. En Asociación Iberoamericana de Educación Superior a Distancia. (2012). Buenas prácticas en educación a distancia. Universidad Técnica Particular de Loja.

Estévez, J.; Castro, J. y Rodríguez, H. (2014). La educación virtual en Colombia. Exposición de modelos de deserción. Institución Universitaria Politécnico Grancolombiano. Recuperado de http://repository.poligran. edu.co/bitstream/10823/570/1/2014.06.24. A R T I C U L O \% 20 E D U C A C I O N \% 20 VIRTUAL.pdf

Facundo Díaz, Á. (2009). Análisis sobre la deserción en la educación superior a distancia y virtual: El caso de la UNADColombia. Revista de Investigaciones UNAD, 8 (2), 117-149. Recuperado de http:// academia.unad.edu.co/images/investigacion/ hemeroteca/revistainvestigaciones/vol.\%208 num._2_2009/An\%C3\% A 11 is is $\% 20$ sobre $\% 201$ a $\% 20$ deserci $\%$ C 3\%B3n\%20 en $\% 201$ a $\% 20$ educaci $\%$ C 3\%B 3 n \% 20 superior $\% 20 \mathrm{a} \% 20 \mathrm{distancia} \% 20 \mathrm{y} \% 20$ virtual $\% 20$ el $\% 20$ caso $\% 20$ de $\% 201 \mathrm{a} \% 20$ unad\%20-\%20colombia.pdf

Florido Bacallao, R. y Florido Bacallao, M. (2003). La Educación a Distancia, sus retos y posibilidades. Revista Eticanet, 1. Recuperado de http://www.ugr.es/ sevimeco/ revistaeticanet/Numero1/Articulos/EaDretos. pdf

García Aretio, L. (2002). La educación a distancia: de la teoría a la práctica. Ariel.

(2001). La educación a 
distancia; ayer y hoy. En Blázquez, Florentino et al. (2001). Sociedad de la información y educación. Junta de Extremadura, Consejería de Educación, Ciencia y Tecnología. Recuperado de http://www.quadernsdigitals. net/datos_web/biblioteca/1_1400/enLinea/0. htm

García, M. (2012).Autorregulación académica como variable explicativa de los procesos de aprendizaje universitario. Profesorado, 16 (1), 203-221. Recuperado de http://digibug.ugr.es/ bitstream/10481/23005/1/rev161ART12.pdf

Garrison, R. (2000). Retos teóricos para la educación a distancia en el siglo XXI. Recuperado de http://especializacion.una.edu. ve/fundamentos/paginas/garrison2000.pdf

González, N. (2003). La educación a distancia, el conocimiento compartido y la creación de una comunidad de discurso internacional. En Beltrán, Daniel y Vásquez, Cesar. (2003). Mediaciones y prácticas pedagógicas en educación a distancia. Bogotá: Universidad Nacional Abierta y a Distancia UNAD.

Gunawardena, Ch. N. \& McIsaac, M. S. (2004). Distance education. In D. H. Jonassen (Ed.), Handbook of Research in Educational Communications and Technology, 2nd ed. (pp. 355-395). Mahwah, NJ: Lawrence Erlbaum Associates. Recuperado de http://ocw.metu. edu.tr/file.php/118/Week10/GunawardenaMcIsaac-distance-ed.pdf

Iriondo, W. y Gallego, D. (2013). El currículo y la educación a distancia. RIED, 16 (1). Recuperado de http://revistas.uned.es/index. $\mathrm{php} / \mathrm{ried} / \mathrm{article} / \mathrm{view} / 2062 / 1938$

Jiminián, Y. (2009). Deserción en la Educación a Distancia: Caso Universidad Abierta para Adultos (UAPA). En Hernández, Ángel; Rama, Claudio; Jiminián, Yanet y Cruz, Magdalena (Edit). Deserción en las instituciones de educación superior a distancia en América Latina y el Caribe. República Dominicana: UAPA.
King, F.; Young, M.; Drivere-Richmond, K. \& Schrader, P. G. (2001). Defining Distance Learning and Distance Education. AACE Journal V 9 (1). Chesapeake: Association for the Advancement of Computing in Education (AACE). Recuperado de http://www.editlib. org/d/17786

Leal Afanador, J. (2013). La Ecología de la formación e-learning en el contexto universitario. En Arboleda, N. y Rama, C. (Ed.). Educación superior a distancia y virtual en Colombia. pp. 65-79. Bogotá: Virtual Educa.

Lozano, F. y Tamez, L. (2014). Retroalimentación formativa para estudiantes de educación a distancia. Revista Iberoamericana de Educación a Distancia RIED, 17 (2), 197-221.

Matallana, O. y Torres, M. (2011). Caracterización de la educación superior a distancia en las universidades colombianas. Revista de investigaciones UNAD, 10, pp. 60-80. Recuperado de http://datateca.unad. edu.co/contenidos/100103/100103_2013_2/ Evaluacion_Nal/Articulo_3.pdf

MinisteriodeEducación Nacional de Colombia. Decreto 1295 de 2010, abril 20, por el cual se reglamenta el registro calificado de que trata la Ley 1188 de 2008 y la oferta y desarrollo de programas académicos de educación superior. Recuperado de http://www.colombiaaprende. edu.co/html/productos/1685/articles-229117 archivo_pdf1.pdf

Moore, J.; Dickson-Deane, C. \& Galyen, K. (2011). E-Learning, online learning, and distance learning environments: Are they the same? Internet and Higher Education, 14. Recuperado de https://scholar.vt.edu/access/ content/group/5deb92b5-10f3-49db-adeb$7294847 \mathrm{f} 1 \mathrm{ebc} / \mathrm{e}-L$ earning $\% 20 \mathrm{Scott} \% 20$ Midkiff.pdf

Moreno, C. (2010). Todo está para hacer, pero no se hace: pensamientos, realidades 
y posibilidades en la educación superior. Bruselas: CESAL.

Parra Arias, J. (2013) Introducción. En Torres Ortiz, J. (comp.) La investigación a distancia en Colombia y América: intercambio de las experiencias en la formación y aprendizaje. Congreso Internacional de Educación a Distancia, Tunja: Universidad Pedagógica y Tecnológica de Colombia.

Pastor, M. (2005). La educación superior a distancia en el nuevo contexto tecnológico del siglo XXI. Revista de la Educación Superior, 34 (4), 77-93. Recuperado de http://www. redalyc.org/pdf/604/60413606.pdf

Ramírez, L. (2012). Retos de la universidad latinoamericana en el siglo XXI: El reto de la ESaD. Revista Calidad en la educación superior. Universidad de Costa Rica, 3 (1), $1-23$.

República de Colombia. Decreto 2412 de 1982, de agosto 19, por el cual se reglamenta, dirige e inspecciona la Educación Abierta y a Distancia y se crea el consejo de Educación Abierta y a Distancia. Diario oficial 36087, Bogotá, 13 de septiembre de 1982. Recuperado de http://www.mineducacion.gov.co/1621/ articles-103523_archivo_pdf.pdf

Decreto 1820 de

1983, de junio 28, por el cual se reglamenta la Educación Superior Abierta y a Distancia. Diario oficial 36298, Bogotá, 21 de julio de 1983. Recuperado de http://www. mineducacion.gov.co/1621/articles-103622 archivo_pdf.pdf

Rivas, R. de (2012). Prólogo. En Asociación Iberoamericana de Educación Superior a Distancia. Buenas prácticas en educación a distancia. Universidad Técnica Particular de Loja.

Rodríguez Ruiz, J. (2013). Prefacio. En Domínguez, Julio y Rama, Claudio (Ed.). (2013). La Educación a Distancia en el Perú.
Perú: Universidad Católica Los Ángeles de Chimbote.

Rodríguez, N. (2014). Fundamentos del proceso educativo a distancia: enseñanza, aprendizaje y evaluación. Revista Iberoamericana de Educación a Distancia RIED, 17 (2), 75-93.

Saba, F. (2003). Distance education theory, methodology, and epistemology: a pragmatic paradigm. En Moore, Michel y Anderson, William. (2003). Handbook of distance education. London: Lawrence Erlbaum Associates.

Salazar, R. y Melo, Á. (2013). Lineamientos Conceptuales de la Modalidad de Educación a Distancia. En Arboleda, Néstor y Rama, Claudio. (Ed.), Educación superior a distancia y virtual en Colombia. pp. 81-111. Bogotá: Virtual Educa.

Sangrá, A. (2002). Los retos de la educación a distancia. Boletín de la Red Estatal de Docencia Universitaria, 2 (3). Recuperado de http://red-u.net/redu/documentos/volumenes/ vol2_n3/vol2_n3_files/articulos/1sangra.pdf

Sarmiento Moreno, L. (2014). La EAD en Colombia, del ayer al mañana. Signos Universitarios: Revista de la Universidad del Salvador, extra 2 (1). Recuperado de http:// p3.usal.edu.ar/index.php/signos/article/ viewFile/2117/2664

Simpson, M. \& Anderson, B. (2012). History and heritage in open, flexible and distance education. Journal of Open, Flexible and Distance Learning, 16 (2). Recuperado de http://journals.akoaotearoa.ac.nz/index.php/ JOFDL/issue/view/19.

Tichapondwa, M. \& Tau, D. (2009). Introducing distance education. Virtual University for the Small States of the Commonwealth. Recuperado de http://www. col.org/sitecollectiondocuments/introducing distance_education.pdf 
Torres, Á. (2004). La educación superior a distancia: entornos de aprendizaje en red. Guadalajara: Universidad Autónoma Metropolitana.

Unesco. (2002). Aprendizaje abierto $\mathrm{y}$ a distancia: consideraciones sobre tendencias, políticas y estrategias. Recuperado de http://unesdoc.unesco.org/ images/0012/001284/128463s.pdf

Zárate, J.; González, A. y Parra, O.. (2010). Educación a distancia: hacia un horizonte de sentido. En Ávila, Patricia. (2010). Educación a distancia: Actores y experiencias. Ecuador: Universidad Técnica Particular de Loja. 